TRANSACTIONS OF THE

AMERICAN MATHEMATICAL SOCIETY

Volume 194, 1974

\title{
A NONLINEAR BOLTZMANN EQUATION IN TRANSPORT THEORY $\left({ }^{1}\right)$
}

\author{
BY
}

C. V. PAO

\begin{abstract}
The method of successive approximations is used to show the existence of a unique solution to a model of a nonlinear Boltzmann equation under the homogeneous boundary and typical initial conditions. An explicit formula in terms of the prescribed functions for the calculation of an approximate solution and its error estimate are given. This formula reveals an interesting analogy between the initial-boundary value problem of the Boltzmann equation and the Cauchy problem for ordinary differential equations. Numerical results for approximate solutions of the problem can be computed by using a computer. The linear Boltzmann equation is considered as a special case and a similar formula for the calculation of approximate solutions is included.
\end{abstract}

1. Introduction. Let $\Omega_{1}, \Omega_{2}$ be bounded domains in the $m$-dimensional Euclidean space $E^{m}$ and let $\partial \Omega_{1}$ be the boundary of $\Omega_{1}$. Consider the following nonlinear integrodifferential equation (usually referred to as the Boltzmann equation):

$$
\begin{array}{r}
\frac{\partial U}{\partial t}+v \cdot \operatorname{grad}_{x} U+f(t, x, v, U)=\int_{\Omega_{2}} g\left(t, x, v, v^{\prime}, U\left(t, x, v^{\prime}\right)\right) d v^{\prime} \\
\quad\left(t \in(0, T], x \in \Omega_{1}, v \in \Omega_{2}\right)
\end{array}
$$

subject to the boundary and initial conditions

$$
\begin{aligned}
& U(t, x, v)=0 \quad\left(t \in(0, T], x \in \partial \Omega_{1}, v \cdot v<0\right), \\
& U(0, x, v)=\phi(x, v) \quad\left(x \in \Omega_{1}, v \in \Omega_{2}\right)
\end{aligned}
$$

where $\nu$ is the outward unit normal vector on $\partial \Omega_{1}$. In general, $f$ and $g$ are prescribed nonlinear functions of the unknown $U \equiv U(t, x, v)$. In case $f, g$ are linear in $U$ then equation (1.1) reduces to the well-known linearized Boltzmann equation

$$
(1.1)^{\prime} \frac{\partial U}{\partial t}+v \cdot \operatorname{grad}_{x} U+\sigma(t, x, v) U=\int_{\Omega_{2}} \sigma_{s}\left(t, x, v, v^{\prime}\right) U\left(t, x, v^{\prime}\right) d v^{\prime}+q(t, x, v) \text {. }
$$

The purpose of this paper is to show the existence of a unique solution to the problem (1.1)-(1.3) and to give a method for the construction of the solution. In

Received by the editors August 17, 1972.

AMS (MOS) subject classifications (1970). Primary 45K05, 82A70, 82A40; Secondary 85A25, 35F 30 .

Key words and phrases. Boltzmann equations, neutron transport problems, initial-boundary value problems, successive approximations, existence and uniqueness of a solution, operator equations.

(1) This work was supported by the U. S. Army Research Office, Durham, North Carolina. 
fact, we present an explicit formula for the calculation of approximate solutions as well as errcr estimates.

The Boltzmann equation (1.1) (or (1.1)') has many applications in the physical sciences. In the theory of neutron transport problem, $U$ represents the neutron's distribution, $\sigma, \sigma_{s}$ are the total and scattering cross-sections and $q$ is the source function. The nonlinear version of (1.1) includes the possibility of collisions among moving neutrons. The boundary condition (1.2) means that no neutrons enter the medium $\Omega_{1}$ through the boundary surface $\partial \Omega_{1}$. (This is the case if, for example, the medium is surrounded by vacuum.) The same mathematical model also describes the time-dependent radiative transfer problem. Literature dealing with the Boltzmann equation is extensive. Some recent results on neutron transport and radiative transfer problems can be found in [2], [9], [10], [13]-[16] and that on kinetic gas theory in [1], [2], [7], [8]. For some earlier work on these subjects the reader is referred to [3]-[6], [11], [12], [17]. Most of the above work on neutron transport and radiative transfer concerned with the linear Boltzmann equation of the form (1.1)'. Some nonlinear transport models have recently been investigated by the author in [13], [14]. In this paper we consider a model suggested by the neutron transport problem and use some of the techniques in [14] for the existence and the method of construction of a classical solution to the problem (1.1)-(1.3).

2. The main results. Let $\Omega=\Omega_{1} \times \Omega_{2}, D=[0, T] \times \Omega_{1} \times \bar{\Omega}_{2}$ and let $C(\bar{D})$ be the set of all (real or complex) functions $u(t, x, v)$ defined on $\bar{D}$ such that (i) $u$ is continuous on $D$, (ii) $u$ is bounded on $\bar{D}$, and (iii) $\lim u(t, x, v)$ exists as $x \rightarrow x_{0} \in \partial \Omega_{1}$ for $t \in[0, T], v \in \bar{\Omega}_{2}$, where $\bar{S}$ denotes the closure of $S$ in $E^{m}$. Define a norm in $C(\bar{D})$ by $\|u\|=\sup \{|u(z)| ; z \in \bar{D}\}$. Then $C(\bar{D})$ is a Banach space. A similar definition holds for the Banach space $C(\bar{\Omega})$ with norm $\|\cdot\|_{\Omega}$. We denote by $C_{x}(\bar{D})$ (resp. $C_{x}(\bar{\Omega})$ ) the set of all functions $u$ in $C(\bar{D})$ such that their first partial derivatives in $x_{1}, \ldots, x_{m}$ (denoted by $u_{x}$ ) are in $C(\bar{D})($ resp. $C(\bar{\Omega})$ ).

By letting $u=e^{-\lambda t} U$ for some real $\lambda>0$ we transform the problem (1.1)-(1.3) to the form

$$
\begin{aligned}
u_{t}+v \cdot \operatorname{grad}_{x} u+\lambda u & =F(t, x, v, u) \quad(t \in(0, T],(x, v) \in \Omega) \\
u(t, x, v) & =0 \quad\left(t \in(0, T], x \in \partial \Omega_{1}, v \cdot \nu<0\right) \\
u(0, x, v) & =\phi(x, v) \quad((x, v) \in \Omega)
\end{aligned}
$$

where $u_{t} \equiv \partial u / \partial t$ and

$$
F(t, x, v, u) \equiv-e^{-\lambda t} f\left(t, x, v, e^{\lambda t} u\right)+\int_{\Omega_{2}} e^{-\lambda t} g\left(t, x, v, v^{\prime}, e^{\lambda t} u\left(t, x, v^{\prime}\right)\right) d v^{\prime}
$$

In the case of the linear equation $(1.1)^{\prime}, F$ is replaced by

$$
F_{0}(t, x, v, u) \equiv-\sigma(t, x, v) u+\int_{\Omega_{2}} \sigma_{s}\left(t, x, v, v^{\prime}\right) u\left(t, x, v^{\prime}\right) d v^{\prime}+e^{-\lambda t} q(t, x, v)
$$


Thus the existence, uniqueness and construction of a solution for the problem (1.1)-(1.3) follow from the same as for the problem (2.1)-(2.3).

Let $u^{(0)} \in C_{x}(\bar{D})$ be given. Define a sequence $\left\{u^{(k)}\right\}$ successively from the following linear problem:

$$
\begin{gathered}
u_{t}^{(k)}+v \cdot \operatorname{grad}_{x} u^{(k)}+\lambda u^{(k)}=F\left(t, x, v, u^{(k-l)}\right), \\
u^{(k)}(t, x, v)=0 \quad\left(t \in(0, T], x \in \partial \Omega_{1}, v \cdot \nu<0\right), \\
u^{(k)}(0, x, v)=\phi(x, v) \quad((x, v) \in \Omega) .
\end{gathered}
$$

Our object is to construct the sequence $\left\{u^{(k)}\right\}$ from (2.6)-(2.8) and to show that this sequence converges to the unique solution of $(2.1)-(2.3)$ in $C(\bar{D})$ for some choice of $\lambda$. To accomplish this we make the following hypotheses:

$\left(\mathrm{H}_{1}\right)$ The functions $f, g$ are continuous in $(t, x, v) \in \bar{D}, v^{\prime} \in \bar{\Omega}_{2}, U \in(-\infty$, $\infty)$; and $\phi$ is in $C_{x}(\bar{\Omega})$.

$\left(\mathrm{H}_{2}\right) f_{x}, g_{x}, f_{U}, g_{U}$ exist and are in $C(\bar{D})$ for each $v^{\prime} \in \bar{\Omega}_{2}$ and each $U$ $\in(-\infty, \infty)$ and $f_{U}, g_{U}$ are uniformly bounded in $U \in(-\infty, \infty)$.

The hypothesis $\left(\mathrm{H}_{2}\right)$,implies that there exist nonnegative functions $\rho(t, x, v)$ and $\rho_{s}\left(t, x, v, v^{\prime}\right)$ satisfying

$$
\sup \left\{\left(\rho(t, x, v)+\int_{\Omega_{2}} \rho_{s}\left(t, x, v, v^{\prime}\right) d v^{\prime}\right) ;(t, x, v) \in \bar{D}\right\} \equiv \bar{\rho}<\infty
$$

such that for all $(t, x, v) \in \bar{D}, v^{\prime} \in \Omega_{2}, U_{1}, U_{2} \in(-\infty, \infty)$

$$
\begin{gathered}
\left|f\left(t, x, v, U_{1}\right)-f\left(t, x, v, U_{2}\right)\right| \leq \rho(t, x, v)\left|U_{1}-U_{2}\right| \\
\left|g\left(t, x, v, v^{\prime}, U_{1}\right)-g\left(t, x, v, v^{\prime}, U_{2}\right)\right| \leq \rho_{s}\left(t, x, v, v^{\prime}\right)\left|U_{1}-U_{2}\right| .
\end{gathered}
$$

Theorem 2.1. Assume that $\left(\mathrm{H}_{1}\right),\left(\mathrm{H}_{2}\right)$ hold. Then given any $u^{(0)} \in C_{x}(\bar{D})$ the sequence $\left\{u^{(k)}\right\}$ defined in (2.6)-(2.8) exists and converges to the unique solution $u(t, x, v)$ of $(2.1)-(2.3)$ for any $\lambda>\bar{\rho}$. Moreover each $u^{(k)}$ is given successively by the formula

$$
\begin{aligned}
u^{(k)}(t, x, v)= & e^{\lambda t} \phi(x-v t, v) \\
& +\int_{0}^{t} e^{-\lambda(t-\tau)} F\left(\tau, x-v(t-\tau), v, u^{(k-1)}(\tau, x-v(t-\tau), v)\right) d \tau, \\
& k=1,2, \ldots,
\end{aligned}
$$

and satisfies the error estimate

$$
\left\|u^{(k)}-u\right\| \leq(\bar{\rho} /(\lambda-\bar{\rho}))(\bar{\rho} / \lambda)^{k-1}\left\|u^{(1)}-u^{(0)}\right\|,
$$

where $F$ is given in (2.4).

In the case of the linear equation (1.1)' the conditions (2.9), (2.10) are satisfied 
with $\rho=\sigma, \rho_{s}=\sigma_{s}$. An immediate consequence of Theorem 2.1 is the following

Corollary. Assume that $\sigma, \sigma_{s}, q$ are in $C_{x}(\bar{D})$ for each $v^{\prime} \in \bar{\Omega}_{2}$ and that $\sigma_{s}$ is continuous on $\bar{D} \times \bar{\Omega}_{2}$. Then by taking $u^{(0)} \in C_{x}(\bar{D})$ and choosing

$$
\lambda>\bar{\sigma} \equiv \sup \left\{\sigma(t, x, v)+\int_{D_{2}} \sigma_{s}\left(t, x, v, v^{\prime}\right) d v^{\prime} ;(t, x, v) \in \bar{D}\right\}
$$

the sequence $\left\{u^{(k)}\right\}$ defined in (2.6)-(2.8) with $F=F_{0}$ exists and converges to the unique solution $u(t, x, v)$ of the linear problem (2.1)-(2.3). In addition, each $u^{(k)}$ can be calculated from (2.11) with $F$ replaced by $F_{0}$ and (2.12) holds with $\bar{\rho}=\bar{\sigma}$.

It is interesting to note that Theorem 2.1 is in analogy to the classical results for the Cauchy problem of ordinary differential equations by the method of successive approximations. The essential conditions in Theorem 2.1 are that $f$ and $g$ satisfy some global Lipschitz conditions. In case they satisfy only local Lipschitz conditions in some neighborhood of the zero function we also have an analogous theorem for the existence of a unique local solution.

Theorem 2.2. Assume that $\left(\mathrm{H}_{1}\right),\left(\mathrm{H}_{2}\right)$ hold only for $U$ in $[-r, r]$ for some $r>0$ and that $\|\phi\|_{\Omega}<r$. Then the problem (1.1)-(1.3) has a unique "local solution" $U(t, x, v)$ in the sense that for some $T_{0}>0, U(t, x, v)$ satisfies (1.1)-(1.3) for $t$ $\in\left[0, T_{0}\right],(x, v) \in \Omega$. In addition, $U$ can be continued for as long as it remains in $(-r, r)$.

We next give some additional conditions for the continuation of a local solution to the whole interval $[0, T]$. For simplicity we consider $C(\bar{D})$ and $C(\bar{\Omega})$ as real spaces of real-valued functions. In addition, we make the following assumption:

$\left(\mathrm{H}_{3}\right)$ For each $(t, x, v) \in \bar{D}, f(t, x, v, 0)=g\left(t, x, v, v^{\prime}, 0\right)=0$ and

$$
U f(t, x, v, U) \geq\left(\int_{D_{2}} \rho_{s}\left(t, x, v, v^{\prime}\right) d v^{\prime}\right) U^{2} \quad(U \in[-r, r]) .
$$

Theorem 2.3. Assume that $\left(\mathrm{H}_{1}\right)-\left(\mathrm{H}_{3}\right)$ hold for $U$ in $[-r, r]$ and that $\|\phi\|_{\Omega}<r$. Then the problem (1.1)-(1.3) has a unique solution $u(t, x, v)$ on the whole interval $[0, T]$. In addition, $\|u(t)\|_{\Omega} \leq\|\phi\|_{\Omega}$ for all $t \in[0, T]$.

It is seen from Theorem 2.3 that if $\left(\mathrm{H}_{1}\right)-\left(\mathrm{H}_{3}\right)$ hold for all $t \in[0, \infty)$ and if the initial function $\phi$ is in $(-r, r)$ then every solution of the problem (1.1)-(1.3) is uniformly bounded for all $t \geq 0$. In the theory of Lyapunov stability, the zero solution of the problem (1.1)-(1.3) (with $\phi=0)$ is stable. In fact, if the condition (2.13) is slightly strengthened by assuming

$$
U f(t, x, v, U) \geq\left(\varepsilon+\int_{D_{2}} \rho_{s}\left(t, x, v, v^{\prime}\right) d v^{\prime}\right) U^{2}
$$

for some $\varepsilon>0$ then the zero solution is (exponentially) asymptotically stable. 
The physical interpretation of (2.13) (or (2.14)) is that if the rate of the particles absorption and scattering by the medium is no less than the rate of particles gained from scattering of other particles then the particle distribution in the medium is nonincreasing (or strictly decreasing).

3. Proof of the theorems. Define an operator $A$ in $C(\bar{D})$ by $A u=u_{t}$ $+v \cdot \operatorname{grad}_{x} u+\lambda u(u \in D(A))$ where $D(A)$ is the domain of $A$ given by $D(A)$ $=\left\{u \in C(\bar{D}) ; u_{t}\right.$ and $v \cdot \operatorname{grad}_{x} u$ are in $C(\bar{D})$ and $u$ satisfies (2.2), (2.3)\}. Consider $F$ as a mapping on $C(\bar{D})$. Then by $\left(\mathrm{H}_{1}\right)$ the problem (2.1)-(2.3) is equivalent to the operator equation

$$
A u=F(u) \quad(u \in D(A))
$$

in the Banach space $C(\bar{D})$, and the problem (2.6)-(2.8) becomes

$$
A u^{(k)}=F\left(u^{(k-1)}\right) \quad\left(u^{(k)} \in D(A)\right) .
$$

Thus it suffices to show that the sequence $\left\{u^{(k)}\right\}$ from (3.2) exists and converges to the unique solution of $(3.1)$ in $C(\bar{D})$. We begin with the following lemma.

Lemma 3.1. Given any $u_{1}, u_{2} \in D(A)$ there exists $z_{0}=\left(t_{0}, x_{0}, v_{0}\right) \in \bar{D}$ such that

$$
\operatorname{Re}\left[\left(\bar{u}_{1}\left(z_{0}\right)-\bar{u}_{2}\left(z_{0}\right)\right)\left(A u_{1}\left(z_{0}\right)-A u_{2}\left(z_{0}\right)\right)\right] \geq \lambda\left\|u_{1}-u_{2}\right\|^{2},
$$

where $\left\|u_{1}-u_{2}\right\|=\left|u_{1}\left(z_{0}\right)-u_{2}\left(z_{0}\right)\right|$. Moreover the inverse $A^{-1}$ exists on $R(A)$, the range of $A$, and

$$
\left\|A^{-1} w_{1}-A^{-1} w_{2}\right\| \leq \lambda^{-1}\left\|w_{1}-w_{2}\right\| \quad\left(w_{1}, w_{2} \in R(A)\right) .
$$

Proof. Let $u=u_{1}-u_{2}$ and let $z_{0} \equiv\left(t_{0}, x_{0}, v_{0}\right) \in \bar{D}$ such that $\|u\|=\left|u\left(z_{0}\right)\right|$. Then

$$
\begin{aligned}
\operatorname{Re}\left[\bar{u}\left(z_{0}\right)(A\right. & \left.\left.u_{1}\left(z_{0}\right)-A u_{2}\left(z_{0}\right)\right)\right] \\
& =\operatorname{Re}\left[\bar{u}\left(z_{0}\right)\left(u_{t}\left(z_{0}\right)+v_{0} \cdot \operatorname{grad}_{x} u\left(z_{0}\right)\right)+\lambda\left|u\left(z_{0}\right)\right|^{2}\right] \\
& =\left(\frac{1}{2} \partial / \partial t\right)\left|u\left(z_{0}\right)\right|^{2}+\frac{1}{2} v_{0} \cdot \operatorname{grad}_{x}\left(\left|u\left(z_{0}\right)\right|^{2}\right)+\lambda\left|u\left(z_{0}\right)\right|^{2} .
\end{aligned}
$$

Since $\left|u\left(z_{0}\right)\right|^{2} \equiv \bar{u}\left(z_{0}\right) u\left(z_{0}\right)$ is a positive maximum on $\bar{D}$, if $t_{0} \in(0, T), x_{0} \in \Omega_{1}$ then $\left|u_{t}\left(z_{0}\right)\right|^{2}=v_{0} \cdot \operatorname{grad}_{x}\left(\left|u\left(z_{0}\right)\right|^{2}\right)=0$ and thus (3.3) follows from (3.5). In case $t_{0}=0$ or $t_{0}=T$ then from $u(0, x, v)=0$ and the continuity of $u, u\left(t_{0}, x_{0}, v_{0}\right)$ $=0$ if $t_{0}=0$; and $(\partial / \partial t)\left(\left|u\left(t_{0}, x_{0}, v_{0}\right)\right|^{2}\right) \geq 0$ if $t_{0}=T$. In either case the first term at the right side of (3.5) is nonnegative. Suppose $x_{0} \in \partial \Omega_{1}$. Then if $v_{0} \cdot \nu<0$, the boundary condition (2.2) implies that $u\left(z_{0}\right)=0$. Since $\left(\mid u\left(t_{0}, x_{0}\right.\right.$, $\left.\left.v_{0}\right)\left.\right|^{2}-\left|u\left(t_{0}, x, v_{0}\right)\right|^{2}\right) /\left|x_{0}-x\right| \geq 0$ for every $x \in \Omega_{1}$, the direction of the vector $\operatorname{grad}_{x}\left(\left|u\left(z_{0}\right)\right|^{2}\right)$ is not pointing inward from $\partial \Omega_{1}$ at $x_{0}$. Hence $v_{0} \cdot \operatorname{grad}_{x}\left(\left|u\left(z_{0}\right)\right|^{2}\right)$ $\geq 0$ if $v_{0} \cdot \nu \geq 0$. This shows that the second term at the right side of (3.5) is also nonnegative. Therefore (3.3) follows from (3.5). Finally, by (3.3) 


$$
\left\|u_{1}-u_{2}\right\|\left\|A u_{1}-A u_{2}\right\| \geq \lambda\left\|u_{1}-u_{2}\right\|^{2},
$$

the second part of the lemma follows.

Lemma 3.2. Given any $h(t, x, v)$ in $C_{x}(\bar{D})$, the linear equation

$$
u_{t}+v \cdot \operatorname{grad}_{x} u+\lambda u=h
$$

under the boundary and initial conditions (2.2), (2.3) has a unique solution $u \in D(A)$ given by

$$
u(t, x, v)=e^{-\lambda t} \phi(x-v t, v)+\int_{0}^{t} e^{-\lambda(t-\tau)} h(\tau, x-v(t-\tau), v) d \tau
$$

where $\phi(x, v)$ and $h(t, x, v)$ are defined to be zero when $x \notin \bar{\Omega}_{1}$.

Proof. By introducing the new variable $x^{\prime}=x-v \tau, t=\tau$, equation (3.7) reduces to

$$
\frac{d}{d \tau} u\left(\tau, x^{\prime}+v \tau, v\right)+\lambda u\left(\tau, x^{\prime}+v \tau, v\right)=h\left(\tau, x^{\prime}+v \tau, v\right) .
$$

Multiplication by $e^{\lambda \tau}$ and integration from 0 to $t$ lead to

$$
e^{\lambda t} u\left(t, x^{\prime}+v t, v\right)-u\left(0, x^{\prime}, v\right)=\int_{0}^{t} e^{\lambda \tau} h\left(\tau, x^{\prime}+v \tau, v\right) d \tau .
$$

By setting $x^{\prime}=x-v t$ and using the initial condition (1.3) we obtain (3.8). A direct calculation from (3.8) shows that $u_{t}$ and $v \cdot \operatorname{grad}_{x} u$ are in $C(\bar{D})$. Since the points $x-v t$ and $x-v(t-\tau)$ are outside of $\bar{\Omega}_{1}$ for all $t>\tau>0$ when $x \in \partial \Omega_{1}$ and $v$ is incoming (i.e., $v \cdot \nu<0)$ we have by definition $\phi(x-v t, v)=h(\tau, x$ $-v(t-r), v)=0$. This shows that $u$ satisfies the boundary condition (2.2). The initial condition (2.3) follows directly from (3.8). Therefore $u \in D(A)$ which proves the lemma.

Lemma 3.2 shows that the sequence $\left\{u^{(k)}\right\}$ defined in (2.6)-(2.8) (or equivalently (3.2)) exists and $u^{(k)} \in D(A)$ for every $k=1,2, \ldots$

Proof of Theorem 2.1. Let $w(t, x, v) \in C_{x}(\bar{D})$ be given and let $h(t, x, v)$ $=F(w)(t, x, v) \equiv F(t, x, v, w(t, x, v))$. Then from $\left(\mathrm{H}_{2}\right), h \in C_{x}(\bar{D})$. Thus by Lemma 3.2 there exists $u \in D(A)$ such that $A u=F(w)$ and by Lemma 3.1 we may write $u=A^{-1} F(w)$. Since from (2.9), (2.10) given any $w_{1}, w_{2} \in C_{x}(\bar{D})$,

$$
\left|F\left(w_{1}\right)(t, x, v)-F\left(w_{2}\right)(t, x, v)\right| \leq\left(\rho(t, x, v)+\int_{D_{2}} \rho_{s}\left(t, x, v, v^{\prime}\right) d v^{\prime}\right)\left\|w_{1}-w_{2}\right\| .
$$

Thus $\left\|F\left(w_{1}\right)-F\left(w_{2}\right)\right\| \leq \bar{\rho}\left\|w_{1}-w_{2}\right\|$. It follows from Lemma 3.1 that

$$
\left\|A^{-1} F\left(w_{1}\right)-A^{-1} F\left(w_{2}\right)\right\| \leq \lambda^{-1}\left\|F\left(w_{1}\right)-F\left(w_{2}\right)\right\| \leq \lambda^{-1} \bar{\rho}\left\|w_{1}-w_{2}\right\| .
$$

This shows that $A^{-1} F$ is a contraction mapping on $C_{x}(\bar{D})$. Since by Lemma 3.1 the sequence $\left\{u^{(k)}\right\}$ determined from (3.2) may be written as $u^{(k)}=A^{-1} F\left(u^{k-1}\right)$, the contraction property of $A^{-1} F$ implies that this sequence converges to a unique solution $u$ (say) in $C(\bar{D})$. But from (3.8) in Lemma 3.2, 


$$
\begin{aligned}
u^{(k)}(t, x, v)= & e^{-\lambda t} \phi(x-v t, v) \\
& +\int_{0}^{t} e^{-\lambda(t-\tau)} F\left(\tau, x-v(t-\tau), v, u^{(k-1)}(\tau, x-v(t-\tau), v)\right) d \tau
\end{aligned}
$$

we see by taking $k \rightarrow \infty$ that $u=A^{-1} F(u)$. Hence $u \in D(A)$ and $A u=F(u)$. This shows the existence of a unique solution to the problem (2.1)-(2.3) and that (2.11) holds. Finally the error estimate follows directly from the contraction property of $A^{-1} F$. The proof of the theorem is complete.

Proof of Theorem 2.2. Let $0<\varepsilon<r-\|\phi\|$. Define extensions $\tilde{f}, \tilde{g}$ in such a way that $\tilde{f}=f, \tilde{g}=g$ for $|U| \leq r-\varepsilon$ and $\tilde{f}, \tilde{g}$ satisfy $\left(\mathrm{H}_{1}\right),\left(\mathrm{H}_{2}\right)$. (For example, we may define $\tilde{f}(t, x, v, U)=f(t, x, v, U)$ if $|U| \leq r-\varepsilon ; \tilde{f}(t, x, v, U)=f(t, x, v, r)$ if $U \geq r ; \tilde{f}(t, x, v, U)=f(t, x, v,-r)$ if $U \leq-r$; and $\tilde{f}$ is continuous on $\bar{D}$ $\times[r-\varepsilon, r]$ and on $\bar{D} \times[-r,-r+\varepsilon]$ such that $f$ is continuously differentiable in $U$ with $\tilde{f}_{U}(t, x, v, \pm r \mp \varepsilon)=f_{U}(t, x, v, \pm r \mp \varepsilon)$ and $\tilde{f}_{U}(t, x, v, \pm r)=0$.) Hence by Theorem 2.1, the extended problem (1.1)-(1.3) with $f, g$ replaced by $\tilde{f}, \tilde{g}$ has a unique solution $\tilde{U}(t, x, v)$. Since $\tilde{U}(t, x, v)$ is continuous in $t$ with $|\tilde{U}(0, x, v)|$ $=|\phi(x, v)|\left\langle r-\varepsilon\right.$ there exists $T_{0}>0$ such that $|\tilde{U}(t, x, v)| \leq r-\varepsilon$ for $t$ $\in\left[0, T_{0}\right],(x, v) \in \bar{\Omega}$. But $\tilde{f}, \tilde{g}$ coincide with $f, g$ when $|\tilde{U}| \leq r-\varepsilon$ we see that $\tilde{U}(t, x, v)$ is the solution of the original problem (1.1)-(1.3) for $t \in\left[0, T_{0}\right]$, $(x, v) \in \bar{\Omega}$, and the solution can be continued so long as it remains in $(-r, r)$. This proves the theorem.

In order to prove Theorem 2.3 we show the following lemma which is useful in the study of the stability problem of a solution.

Lemma 3.3. For each $t \in[0, T)$ let $u(t, x, v) \in C(\bar{\Omega})$ such that $u_{t}(t, x, v)$ exists and is in $C(\bar{\Omega})$. Then there exists $z_{0} \equiv\left(x_{0}, v_{0}\right) \in \bar{\Omega}\left(x_{0}, v_{0}\right.$ may depend on $\left.t\right)$ with $\|u(t)\|_{\Omega}=\left|u\left(t, z_{0}\right)\right|$ such that the right derivative $\left(d^{+} / d t\right)\left|u\left(t, z_{0}\right)\right|$ exists and satisfies

$$
\|u(t)\|_{\Omega}\left(d^{+} / d t\right)\left|u\left(t, z_{0}\right)\right| \leq u\left(t, z_{0}\right) u_{t}\left(t, z_{0}\right) .
$$

Proof. We first show that for each fixed $z \equiv(x, v) \in \bar{\Omega},\left(d^{+} / d t\right)|u(t, z)|$ exists and

$$
\left(d^{+} / d t\right)|u(t, z)|=\lim _{h \rightarrow 0^{+}} h^{-1}\left(\left|u(t, z)+h u_{t}(t, z)\right|-|u(t, z)|\right) .
$$

The limit in (3.11) exists since the function $g(h) \equiv h^{-1}\left(\left|u(t, z)+h u_{t}(t, z)\right|\right.$ $-|u(t, z)|)$ is monotonically nondecreasing in $h$ and is bounded by $\pm\left|u_{t}(t, z)\right|$. From the relation

$$
\begin{gathered}
h^{-1}\left|(|u(t+h, z)|-|u(t, z)|)-\left(\left|u(t, z)+h u_{t}(t, z)\right|-|u(t, z)|\right)\right| \\
\leq h^{-1}\left|u(t+h, z)-u(t, z)-h u_{t}(t, z)\right|
\end{gathered}
$$

for every $h>0$ we see by taking $h \rightarrow 0^{+}$that (3.11) holds. Let $S_{t}=\{z$ $\left.\in \bar{\Omega} ;\|u(t)\|_{\Omega}=|u(t, z)|\right\}$. Then by taking any $z_{0} \in S_{t}$ in (3.11) we have

$$
\left(d^{+} / d t\right)\left|u\left(t, z_{0}\right)\right| \leq \lim _{h \rightarrow 0^{+}}\left(\left\|u(t)+h u_{t}(t)\right\|_{\Omega}-\|u(t)\|_{\Omega}\right) \quad(t \in[0, T)) .
$$


Notice that the limit in (3.12) also exists. Since (3.10) holds for $\|u(t)\|_{\Omega}=0$ we need only to show the case $u(t, z) \neq 0$. For convenience we surpress the fixed $t$ in $u(t, z)$.

For each $h>0$ we choose $z_{h} \in \bar{\Omega}$ such that $\left\|u+h u_{t}\right\|_{\Omega}=\left|u\left(z_{h}\right)+h u_{t}\left(z_{h}\right)\right|$. Then for any $z^{*} \in S_{t}$

$$
\left|u\left(z^{*}\right)+h u_{t}\left(z^{*}\right)\right| \leq\left\|u+h u_{t}\right\|_{\Omega}=\left|u\left(z_{h}\right)+h u_{t}\left(z_{h}\right)\right| .
$$

Since $\left\{z_{h}\right\}$ is bounded we may assume that $z_{h} \rightarrow z_{0}$ (say). Letting $h \rightarrow 0^{+}$in (3.13) gives $\left|u\left(z^{*}\right)\right| \leq\left|u\left(z_{0}\right)\right|$. Thus $z_{0} \in S_{t}$. Now if $u\left(z_{0}\right) \gtrless 0$ then for sufficiently small $h, u\left(z_{h}\right) \gtrless 0$ and $u\left(z_{h}\right)+h u_{t}\left(z_{h}\right) \gtrless 0$ so that

$$
h^{-1}\left(\left|u\left(z_{h}\right)+h u_{t}\left(z_{h}\right)\right|-\left|u\left(z_{h}\right)\right|\right)=\left(\operatorname{sgn} u\left(z_{0}\right)\right) u_{t}\left(z_{h}\right) .
$$

But for each $h>0$,

$$
h^{-1}\left(\left|u\left(z_{h}\right)+h u_{t}\left(z_{h}\right)\right|-\left|u\left(z^{*}\right)\right|\right) \leq h^{-1}\left(\left|u\left(z_{h}\right)+h u_{t}\left(z_{h}\right)\right|-\left|u\left(z_{h}\right)\right|\right) .
$$

We obtain from (3.14), (3.15) that

$$
\lim _{h \rightarrow 0^{+}} h^{-1}\left(\left\|u+h u_{t}\right\|_{\Omega}-\|u\|_{\Omega}\right) \leq \frac{u\left(z_{0}\right)}{\|u\|} u_{t}\left(z_{0}\right)
$$

Hence (3.10) follows from (3.12) and (3.16).

Remark. From the boundedness of $u_{t}$ on $\bar{D}$, there exists a constant $M<\infty$ such that $|u(t, z)-u(s, z)| \leq M|t-s|$ for all $t, s \in[0, T], z \in \bar{\Omega}$. Hence $\|u(t)\|_{\Omega}$ is Lipschitz continuous in $t$ and thus is differentiable almost everywhere in $[0, T]$. This implies that (3.10) holds for $(d / d t)\left|u\left(t, z_{0}\right)\right|$ for almost all values of $t \in[0, T]$.

Proof of Theorem 2.3. By defining extensions $\tilde{f}, \tilde{g}$ as in the proof of Theorem 2.2, the extended problem (1.1)-(1.3) has a unique solution $\tilde{U}(t, x, v) \in D(A)$. By Lemma 3.3 there exists $z_{0} \equiv\left(x_{0}, v_{0}\right) \in \bar{\Omega}$ such that

$$
\begin{aligned}
\|\tilde{U}(t)\|_{\Omega}\left(d^{+} / d t\right)\left|\tilde{U}\left(t, z_{0}\right)\right| & \\
\leq \tilde{U}\left(t, z_{0}\right)\left[-v_{0} \cdot \operatorname{grad}_{x} \tilde{U}\left(t, z_{0}\right)\right. & -\tilde{f}\left(t, z_{0}, \tilde{U}\left(t, z_{0}\right)\right) \\
& \left.+\int_{\Omega_{2}} \tilde{g}\left(t, z_{0}, v^{\prime}, \tilde{U}\left(t, x_{0}, v^{\prime}\right)\right) d v^{\prime}\right]
\end{aligned}
$$

where $\|\tilde{U}(t)\|_{\Omega}=\left|\tilde{U}\left(t, z_{0}\right)\right|$. From the proof of Lemma 3.1 we see that $\dot{U}\left(t, z_{0}\right)$. $\left(v_{0} \cdot \operatorname{grad}_{x} \tilde{U}\left(t, z_{0}\right)\right) \geq 0$. Since $\tilde{g}$ satisfies (2.10), the right side of (3.17) is majorized by

$$
-\tilde{U}\left(t, z_{0}\right) \tilde{f}\left(t, z_{0}, \tilde{U}\left(t, z_{0}\right)\right)+\left(\int_{\Omega_{2}} \rho_{s}\left(t, z_{0}, v^{\prime}\right) d v^{\prime}\right)\left|\tilde{U}\left(t, z_{0}\right)\right|^{2}
$$

It follows from (3.17), $\left(\mathrm{H}_{3}\right)$ and the continuity of $\left|\tilde{U}\left(t, z_{0}\right)\right|$ that

$$
\left(d^{+} / d t\right)\left|\tilde{U}\left(t, z_{0}\right)\right|^{2} \leq 0 \text { for as long as }\|\tilde{U}(t)\| \leq r .
$$


Since this is true for every $t \in[0, T)$ and a corresponding point $z_{0}$ in $\bar{\Omega}$ we have

$$
\|\tilde{U}(t)\|_{\Omega} \leq\|\tilde{U}(0)\|_{\Omega}=\|\phi\|_{\Omega}<r \text { for all } t \in[0, T] .
$$

But $\tilde{f}, \tilde{g}$ coincide with $f, g$, respectively, when $\|\tilde{U}(t)\|<r$ we conclude that $\tilde{U}(t, x, v)$ is the solution of the original problem (1.1)-(1.3). This proves the theorem.

In case (2.13) is replaced by (2.14) then (3.18) becomes

$$
\left(d^{+} / d t\right)\left|\tilde{U}\left(t, z_{0}\right)\right|^{2} \leq-2 \varepsilon\|\tilde{U}(t)\|_{\Omega}^{2}
$$

and thus $\|\tilde{U}(t)\|_{\Omega} \leq e^{-\varepsilon t}\|\phi\|_{\Omega}$ for all $t$. This justifies the remark following Theorem 2.3.

\section{REFERENCES}

1. L. Arkeryd, An existence theorem for a modified space-inhomogeneous, nonlinear Boltzmann equation, Bull. Amer. Math. Soc. 78 (1972), 610-614. MR 45 \#8195.

2. R. Bellman, G. Birkhoff and I. Abu-Shumays (Editors), Transport theory, SIAM-AMS Proceedings, Vol. 1, Amer. Math. Soc., Providence, R. I., 1969.

3. G. Birkhoff, Positivity and criticality, Proc. Sympos. Appl. Math., vol. 11, Amer. Math. Soc., Providence, R. I., 1961, pp. 116-126. MR 22 \#11563.

4. S. Chandrasekhar, Radiative transfer, Dover, New York, 1960. MR 22 \#2446.

5. B. Davison and J. B. Sykes, Neutron transport theory, Clarendon Press, Oxford, 1957. MR 20 \#2217.

6. H. Grad, Principles of the kinetic theory of gases, Handbuch der Physik (herausgegeben von S. Flügge), Band 12, Thermodynamik der Gase, Springer-Verlag, Berlin, 1958, pp. 205-294. MR 24 \# B1583.

7.—, Asymptotic equivalence of the Navier-Stokes and nonlinear Boltzmann equations, Proc. Sympos. Appl. Math., vol. 17, Amer. Math. Soc., Providence, R. I., 1965, pp. 154-183. MR 32 \# 1979.

8. F. A. Grünbaum, Linearization for the Boltzmann equation, Trans. Amer. Math. Soc. 165 (1972), 425-449. MR 45 \#4783.

9. H. Hejtmanek, Perturbational aspects of the linear transport equation, J. Math. Anal. Appl. 30 (1970), 237-245. MR 41 \#2333.

10. E. Inönü and P. E. Zweifel, Developments in transport theory, Academic Press, New York, 1967.

11. M. Kac, Foundations of kinetic theory, Proc. Third Berkeley Sympos. on Math. Statist. and Probability, 1954/55, vol. III, Univ. of California Press, Berkeley and Los Angeles, 1956, pp. 171-197. MR 18, 960.

12. J. Lehner and G. M. Wing, On the spectrum of an unsymmetric operator arising in the transport theory of neutrons, Comm. Pure Appl. Math 8 (1955), 217-234. MR 16, 1120.

13. C. V. Pao, On nonlinear neutron transport equations, J. Math. Anal. Appl. 42 (1973), 578-593.

14. - Solution of a nonlinear Boltzmann equation for neutron transport in $L^{1}$ space, Arch. Rational Mech. Anal. 50 (1973), 290-302.

15. A. Suhadolc, Linearized Boltzmann equation in $L^{1}$ space, J. Math. Anal. Appl. 35 (1971), 1-13. MR 44 \#3635.

16. I. Vidar, Spectra of perturbed semi-groups with applications to transport theory, J. Math. Anal. Appl. 30 (1970), 264-279. MR 41 \#4297.

17. G. M. Wing, An introduction to transport theory, Wiley, New York, 1962. MR 27 \#5580.

Department of Mathematics, North Carolina State University, Raleigh, North CaroLINA 27607 